\title{
Electromagnetic Scattering of Tubular Cylindrical Structure-Double Series Formulation and Some Results
}

\author{
HUNG-MOU LEE
}

\begin{abstract}
Depending on its length-to-diameter ratio, the tubular cylindrical structure can represent either a tubular cylinder or a strip loop. The problem of electromagnetic scattering from such a structure is studied. Chebyshev polynomials are used to represent the axial dependence of the surface current distribution. Formulation is derived for solving the expansion coefficients of the current distribution. Since the double series expansion coefficients of the Green's function over such a basis are known, no numerical integration is required under this formalism. The theory is applied to compute the backscattering cross section of a tubular cylinder over a range of frequencies. The theoretical results agree with experimental data.
\end{abstract}

\section{INTRODUCTION}

$\mathrm{T}$ HE FINITE TUBULAR cylindrical structure is an interesting geometry for modeling electromagnetic problems. Under the assumption of zero wall thickness, it conforms to the cylindrical coordinate with a constant radius. The rotational symmetry around the axis of the cylinder reduces the problem to a single variable. This geometry contains two parameters: the length and the radius. By assuming different parametric values, this structure serves as a model for the linear antenna [1], [2], the loop [3], the thick antenna [4], [5] and, among other objects, the fuselage [6] and the air intake cavity of a jet [7].

The solution to the problem of electromagnetic scattering from an infinite cylinder is well known [8]. Because of the translational symmetry along the axis of the cylinder, Fourier transformation can be utilized to remove the axial dependence of the Green's function in the infinite cylinder problem. Deprived of this symmetry, a double series expansion for the Green's function is required to solve the finite cylinder problem [2]. With the choice of a complete orthogonal basis, the Chebyshev polynomials of the first kind, analytic expressions for such double series expansion coefficients have been found [9]. This progress makes it very attractive to formulate solutions to electromagnetic problems on the finite cylindrical structure in terms of the surface currents expanded over this basis. Even though closed-form solution to the current distribution is not available and coupled linear equations have to be solved to obtain the final answer, the coefficients of this system of linear equations are determined exactly and can be

Manuscript received January 28, 1986; revised July 23, 1986. This work was supported by the Office of Naval Research under Contract N00014-86WR24009.

The author is with the Department of Electrical and Computer Engineering, Naval Postgraduate School (Code 62Lh), Monterey, CA 93943.

IEEE Log Number 8613198. evaluated efficiently without resorting to numerical integration.

In what follows, the scattered far field in terms of the expansion coefficients of the surface current is also derived. The backscattering cross section along the axis of a tubular cylinder is computed for two cylinders over a range of frequencies. Their circumferences vary from 0.875 to 3.125 wavelengths and their lengths vary roughly from 1.114 to 3.977 wavelengths for one, and from 1.671 to 5.968 wavelengths for the other. The results agree with experimentally measured data.

\section{The Scattering Current}

In this section, formulas will be derived for the sum of the scattering current on the surface of the tubular cylindrical structure excited by an external field. The cylindrical structure can be divided into two cases. The loop case, in which the height of the tubular cylinder is denoted by $2 w$ and the radius is customarily denoted by $b$, satisfies the relation $b>w$. The cylinder case, in which the height of the tubular cylinder is conventionally denoted by $2 h$ and its radius by $2 a$, satisfies the relation $h>a$. To treat both cases at the same time, the electrical length of these parameters will be denoted by $l_{1}=$ $k h$ and $l_{2}=k a$ for the cylinder case and $l_{1}=k w$ and $l_{2}=k b$ for the loop case as shown in Fig. 1 where $k=2 \pi \lambda$. Furthermore, the cylindrical coordinate is scaled so that the cylindrical structure is described by $\rho=1,-1 \leq z \leq 1$.

Assume that the sum of the surface current on the inside and the outside surface of the cylinder is

$$
\mathbf{K}(\phi, z)=K_{\phi}(\phi, z) \hat{\phi}+K_{z}(\phi, z) \hat{\mathbf{z}} .
$$

By applying the Stratton-Chu integral [10] to the surface of the cylinder, the radiated electric fields can be obtained. In the coordinate shown in Fig. 2, they are

$$
\begin{aligned}
& -\frac{2 i}{l_{1} l_{2} \zeta_{0}} E_{\rho}(\rho, \phi, z) \\
& =\frac{1}{l_{1} l_{2}} \frac{\partial^{2}}{\partial z \partial \rho} \int_{-1}^{1} d z_{0} \int_{-\pi}^{\pi} \frac{d \phi_{0}}{2 \pi} G\left(\mathbf{r}-\mathbf{r}_{0}\right) K_{z}\left(\phi_{0}, z_{0}\right) \\
& \quad+\frac{1}{l_{2}^{2}} \frac{\partial^{2}}{\partial \phi \partial \rho} \int_{-1}^{1} d z_{0} \int_{-\pi}^{\pi} \frac{d \phi_{0}}{2 \pi} G\left(\mathbf{r}-\mathbf{r}_{0}\right) K_{\phi}\left(\phi_{0}, z_{0}\right) \\
& \quad+\int_{-1}^{1} d z_{0} \int_{-\pi}^{\pi} \frac{d \phi_{0}}{2 \pi} G\left(\mathbf{r}-\mathbf{r}_{0}\right) K_{\phi}\left(\phi_{0}, z_{0}\right)
\end{aligned}
$$




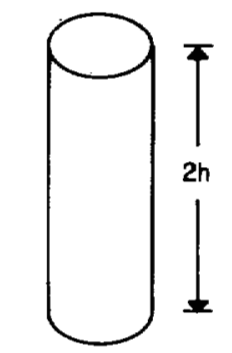

$-42 a$

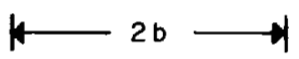

h > a

The Cylinder Cose

$$
\begin{aligned}
& l_{1}=\mathrm{kh} \\
& l_{2}=\mathrm{ka}
\end{aligned}
$$$$
w<b
$$

The Loop Case

$$
l_{1}=\mathrm{kw}
$$$$
l_{2}=k b
$$

Fig. 1. Tubular cylindrical conductors.

$$
\begin{aligned}
& -\frac{2 i}{l_{1} l_{2} \zeta_{0}} E_{\phi}(\rho, \phi, z) \\
& =\frac{1}{l_{1} l_{2} \rho} \frac{\partial^{2}}{\partial \phi \partial z} \int_{-1}^{1} d z_{0} \int_{-\pi}^{\pi} \frac{d \phi_{0}}{2 \pi} G\left(\mathbf{r}-\mathbf{r}_{0}\right) K_{z}\left(\phi_{0}, z_{0}\right) \\
& \quad+\frac{1}{l_{2}^{2} \rho} \frac{\partial^{2}}{\partial \phi^{2}} \int_{-1}^{1} d z_{0} \int_{-\pi}^{\pi} \frac{d \phi_{0}}{2 \pi} G\left(\mathbf{r}-\mathbf{r}_{0}\right) K_{\phi}\left(\phi_{0}, z_{0}\right) \\
& \quad+\int_{-1}^{1} d z_{0} \int_{-\pi}^{\pi} \frac{d \phi_{0}}{2 \pi} G\left(\mathbf{r}-\mathbf{r}_{0}\right) \cos \left(\phi-\phi_{0}\right) K_{\phi}\left(\phi_{0}, z_{0}\right)
\end{aligned}
$$

$$
\begin{aligned}
& -\frac{2 i}{l_{1} l_{2} \zeta_{0}} E_{z}(\rho, \phi, z) \\
& \quad=\left(1+\frac{1}{l_{1}^{2}} \frac{\partial^{2}}{\partial z^{2}}\right) \int_{-1}^{1} d z_{0} \int_{-\pi}^{\pi} \frac{d \phi_{0}}{2 \pi} G\left(\mathbf{r}-\mathbf{r}_{0}\right) K_{z}\left(\phi_{0}, z_{0}\right) \\
& \quad+\frac{1}{l_{1} l_{2}} \frac{\partial^{2}}{\partial \phi \partial z} \int_{-1}^{1} d z_{0} \int_{-\pi}^{\pi} \frac{d \phi_{0}}{2 \pi} G\left(\mathbf{r}-\mathbf{r}_{0}\right) K_{\phi}\left(\phi_{0}, z_{0}\right)
\end{aligned}
$$

where

$$
\begin{gathered}
G\left(\mathbf{r}-\mathbf{r}_{0}\right)=\frac{e^{i k\left|\mathbf{r}-\mathbf{r}_{0}\right|}}{k\left|\mathbf{r}-\mathbf{r}_{0}\right|} \\
\left(k\left|\mathbf{r}-\mathbf{r}_{0}\right|\right)^{2}=l_{2}^{2}\left(\rho^{2}+1-2 \rho \cos \left(\phi-\phi_{0}\right)\right)+l_{1}^{2}\left(z-z_{0}\right)^{2}
\end{gathered}
$$

and

$$
\zeta_{0}=\left(\frac{\mu_{0}}{\epsilon_{0}}\right)^{1 / 2}
$$
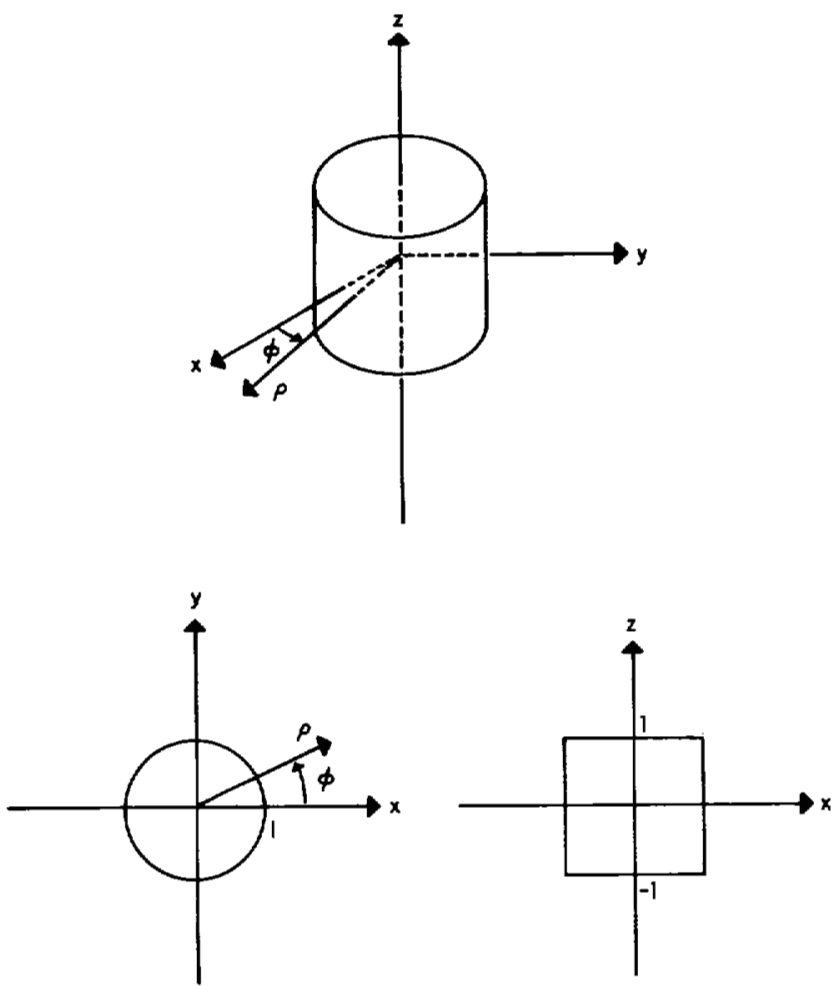

Fig. 2. Cylindrical coordinate system for both the cylinder and the loop cases.

An external field having the tangential components $E_{\phi}^{i}(\phi, z)$ and $E_{z}^{i}(\phi, z)$ on the surface of the cylinder, $\rho=1$ and $-1<$ $z<1$, will excite a scattering surface current. A pair of integrodifferential equations for the surface current can be obtained from (3) and (4) by applying the boundary condition on the cylinder surface:

$$
\begin{aligned}
& \frac{2 i}{l_{1} l_{2} \zeta_{0}} E_{\phi}^{i}(\phi, z) \\
& =\frac{1}{l_{1} l_{2}} \frac{\partial^{2}}{\partial \phi \partial z} \int_{-1}^{1} d z_{0} \int_{-\pi}^{\pi} \frac{d \phi_{0}}{2 \pi} G\left(\mathbf{r}-\mathbf{r}_{0}\right) K_{z}\left(\phi_{0}, z_{0}\right) \\
& \quad+\frac{1}{l_{2}^{2}} \frac{\partial^{2}}{\partial \phi^{2}} \int_{-1}^{1} d z_{0} \int_{-\pi}^{\pi} \frac{d \phi_{0}}{2 \pi} G\left(\mathbf{r}-\mathbf{r}_{0}\right) K_{\dot{\phi}}\left(\phi_{0}, z_{0}\right) \\
& \quad+\int_{-1}^{1} d z_{0} \int_{-\pi}^{\pi} \frac{d \phi_{0}}{2 \pi} G\left(\mathbf{r}-\mathbf{r}_{0}\right) \cos \left(\phi-\phi_{0}\right) K_{\phi}\left(\phi_{0}, z_{0}\right)
\end{aligned}
$$

$\frac{2 i}{l_{1} l_{2} \zeta_{0}} E_{z}^{i}(\phi, z)$

$$
\begin{aligned}
= & \left(1+\frac{1}{l_{1}^{2}} \frac{\partial^{2}}{\partial z^{2}}\right) \int_{-1}^{1} d z_{0} \int_{-\pi}^{\pi} \frac{d \phi_{0}}{2 \pi} G\left(\mathbf{r}-\mathbf{r}_{0}\right) K_{z}\left(\phi_{0}, z_{0}\right) \\
& +\frac{1}{l_{1} l_{2}} \frac{\partial^{2}}{\partial \phi \partial z} \int_{-1}^{1} d z_{0} \int_{-\pi}^{\pi} \frac{d \phi_{0}}{2 \pi} G\left(\mathbf{r}-\mathbf{r}_{0}\right) K_{\phi}\left(\phi_{0}, z_{0}\right) .
\end{aligned}
$$

Because of the rotational symmetry of this problem, Fourier series expansion can be utilized to eliminate the $\phi$-dependence. 
By considering the edge condition [11] for the scattering current at the ends of the cylinder, the following series representation for the surface current is adopted

$$
\begin{gathered}
K_{z}(\phi, z)=\sum_{n=-\infty}^{\infty} e^{i n \phi} K_{z n}(z) \\
=\frac{1}{\pi} \sum_{n=-\infty}^{\infty} e^{i n \phi} \sum_{p=0}^{\infty} K_{z n}^{p} \sin (p+1) v \\
K_{\phi}(\phi, z)=\sum_{n=-\infty}^{\infty} e^{i n \phi} K_{\phi n}(z) \\
=\frac{1}{\pi \sin v} \sum_{n=-\infty}^{\infty} e^{i n \phi} \sum_{p=0}^{\infty} K_{\phi n}^{p} \cos p v
\end{gathered}
$$

where $\cos v=z, 0<v<\pi$.

Note that the edge condition is satisfied term by term in the above expressions.

Equations (7) and (8) can be converted into two sets of linear equations for the expansion coefficients $K_{\phi n}^{p}$ and $K_{z n}^{p}$ of (9) and (10). Since the component $E_{\phi}$ of the scattered field given by the right side of (7) vanishes at the ends of the cylinder while the scattered $E_{z}$ given by the right side of (8) may possess discontinuities as suggested by the edge condition [11], these equations may not be satisfied at $z= \pm 1$. In addition to this difficulty, the $z$-derivatives in (7) and (8) introduce apparent singularities which are not integrable in the variable $v=\cos ^{-1} z$. Thus Fourier series expansion in $v$ cannot be applied directly to solve these equations. Therefore, the factors $\sin v$ and $\sin ^{3} v$ are multiplied to (7) and (8), respectively, before these two equations are expanded over the complete set of orthogonal functions $\{\sin (p+1) v\}, p=0$, $1, \cdots$ on $0 \leq v \leq \pi$. The resulting equations are

$$
\begin{gathered}
\sum_{q=0}^{\infty}\left\{M_{z z n}^{2 p+1,2 q+1} K_{z n}^{2 q+1}+\frac{i n}{l_{2}} M_{z \phi n}^{2 p+1,2 q} K_{\phi n}^{2 q}\right\}=E_{z n}^{2 p+1} \\
\sum_{q=0}^{\infty}\left\{\frac{i n}{l_{2}} M_{\phi z n}^{2 p, 2 q+1} K_{z n}^{2 q+1}+M_{\phi \phi n}^{2 p, 2 q} K_{\phi n}^{2 q}\right\}=E_{\phi n}^{2 p}
\end{gathered}
$$

for $K_{z n}^{2 p+1}, K_{\phi n}^{2 p}$, and

$$
\begin{gathered}
\sum_{q=0}^{\infty}\left\{M_{z z n}^{2 p, 2 q} K_{z n}^{2 q}+\frac{i n}{l_{2}} M_{z \phi n}^{2 p, 2 q+1} K_{\phi n}^{2 q+1}\right\}=E_{z n}^{2 p} \\
\sum_{q=0}^{\infty}\left\{\frac{i n}{l_{2}} M_{\phi z n}^{2 p+1,2 q} K_{z n}^{2 q}+M_{\phi \phi n}^{2 p+1,2 q+1} K_{\phi n}^{2 q+1}\right\}=E_{\phi n}^{2 p+1}
\end{gathered}
$$

for $K_{z n}^{2 p}, K_{\phi n}^{2 p+1}$, where

$E_{z n}^{p}=\frac{32 i}{l_{1} l_{2} \zeta_{0}} \int_{0}^{\pi} \frac{d v}{\pi} \sin ^{3} v \sin (p+1) v$

$$
\cdot \int_{-\pi}^{\pi} \frac{d \phi}{2 \pi} e^{-i n \phi} E_{z}^{i}(\phi, \cos v)
$$

$E_{\phi n}^{p}=\frac{4 i}{l_{1} l_{2} \zeta_{0}} \int_{0}^{\pi} \frac{d v}{\pi} \sin v \sin (p+1) v$

$$
\cdot \int_{-\pi}^{\pi} \frac{d \phi}{2 \pi} e^{-i n \phi} E_{\phi}^{i}(\phi, \cos v)
$$

The coefficients of the linear equations (11)-(14) are

$$
\begin{aligned}
M_{z z n}^{p, q}= & \left(G_{n}^{p+4, q}-G_{n}^{p+4, q+2}\right)-\left(G_{n}^{p-2, q}-G_{n}^{p-2, q+2}\right) \\
& -\left(\frac{4 p(p-1)}{l_{1}^{2}}-3\right)\left(G_{n}^{p, q}-G_{n}^{p, q+2}\right) \\
& +\left(\frac{4(p+2)(p+3)}{l_{1}^{2}}-3\right)\left(G_{n}^{p+2, q}-G_{n}^{p+2, q+2}\right)
\end{aligned}
$$

$M_{z \phi n}^{p, q}=\frac{4}{l_{1}}\left(2(p+1) G_{n}^{p+1, q}\right.$

$$
\left.-(p-1) G_{n}^{p-1, q}-(p+3) G_{n}^{p+3, q}\right)
$$

$M_{\phi z n}^{p, q}=\frac{(p+1)}{l_{1}}\left(G_{n}^{p+1, q}-G_{n}^{p+1, q+2}\right)$

$$
\begin{aligned}
M_{\phi \phi n}^{p, q}=\frac{1}{2}\left(\left(G_{n-1}^{p, q}-G_{n-1}^{p+2, q}\right)+\left(G_{n+1}^{p, q}-G_{n+1}^{p+2, q}\right)\right) & \\
& -\frac{n^{2}}{l_{2}^{2}}\left(G_{n}^{p, q}-G_{n}^{p+2, q}\right) .
\end{aligned}
$$

In the above expressions, $G_{n}^{p, q}$ is the double series expansion coefficient of the Green's function, (5), over the Chebyshev polynomials, after the Fourier series expansion is carried out

$$
\begin{array}{r}
G_{n}^{p, q=} \int_{-1}^{1} \frac{d z}{\pi}\left(1-z^{2}\right)^{-1 / 2} T_{p}(z) \int_{-1}^{1} \frac{d z_{0}}{\pi}\left(1-z_{0}^{2}\right)^{-1 / 2} T_{q}\left(z_{0}\right) \\
\cdot \int_{-\pi}^{\pi} \frac{d \phi}{2 \pi} e^{-i n\left(\phi-\phi_{0}\right)} G\left(\mathbf{r}-\mathbf{r}_{0}\right)
\end{array}
$$

where $T_{p}(\cos v)=\cos p v$ is the Chebyshev polynomial of the first kind of order $p$. Note that for $p, q \geq 0$,

$$
G_{n}^{-p,-q}=G_{n}^{-p, q}=G_{n}^{p,-q}=G_{n}^{p, q}
$$

and for $n \geq 0, G_{-n}^{p, q}=G_{n}^{p, q}$.

Analytic expressions for the above triple integral are available [9] in terms of series sums of the variables $l_{1}$ and $l_{2}$ for both the cylinder case, $l_{1}>l_{2}$, and the loop case, $l_{1}<l_{2}$. These expressions converge to a single value when $l_{1}=l_{2}$. They are reformulated in the forms computer programs for summing up the series are written, which are somewhat different from their original forms [9], and are included in the appendixes.

Since $G_{n}^{p, q}=G_{-n}^{p, q}$, advantage may be taken by separating the surface current into even and odd $\phi$-dependent parts. 
Define:

$$
\begin{gathered}
K_{z n}^{( \pm), p}=K_{z n}^{p} \pm K_{z,-n}^{p} ; K_{\phi n}^{( \pm), p}=K_{\phi n}^{p} \pm K_{\phi,-n}^{p}, \quad \text { for } n>0, \\
K_{z_{0}}^{(+), p}=K_{z 0}^{p} ; K_{\phi_{0}}^{(+), p}=K_{\phi_{0}}^{p} ; K_{z_{0}}^{(-), p}=K_{\phi_{0}}^{(-), p}=0
\end{gathered}
$$

and similarly,

$$
\begin{gathered}
E_{z n}^{( \pm), p}=E_{z n}^{p} \pm E_{z_{1}, n}^{p} ; E_{\phi n}^{( \pm), p}=E_{\phi n}^{p} \pm E_{\phi,-n}^{p}, \quad \text { for } n>0 \\
E_{z_{0}}^{(+), p}=E_{z_{0}}^{p} ; E_{\phi_{0}}^{(+), p}=E_{\phi_{0}}^{p} ; E_{z_{0}}^{(-), p}=E_{\phi_{0}}^{(-), p}=0
\end{gathered}
$$

then (11)-(14) become

$\sum_{q=0}^{\infty}\left\{M_{z z n}^{2 p+1,2 q+1} K_{z n}^{( \pm), 2 q+1}+\frac{i n}{l_{2}} M_{z \phi n}^{2 p+1,2 q} K_{\phi n}^{(\mp), 2 q}\right\}=E_{z n}^{( \pm), 2 p+1}$

$\sum_{q=0}^{\infty}\left\{\frac{\text { in }}{l_{2}} M_{\phi z n}^{2 p, 2 q+1} K_{z n}^{( \pm), 2 q+1}+M_{\phi \phi n}^{2 p, 2 q} K_{\phi n}^{(\mp), 2 q}\right\}=E_{\phi n}^{(\mp), 2 p}$

and

$\sum_{q=0}^{\infty}\left\{M_{z z n}^{2 p, 2 q} K_{z n}^{( \pm), 2 q}+\frac{i n}{l_{2}} M_{z \phi n}^{2 p, 2 q+1} K_{\phi n}^{(\mp), 2 q+1}\right\}=E_{z n}^{( \pm), 2 p}$

$\sum_{q=0}^{\infty}\left\{\frac{i n}{l_{2}} M_{\phi z n}^{2 p+1,2 q} K_{z n}^{( \pm), 2 q}+M_{\phi \phi n}^{2 p+1,2 q+1} K_{\phi n}^{(\mp), 2 q+1}\right\}=E_{\phi n}^{(\mp), 2 p+1}$.

Only the sets of equations with $n \geq 0$ have to be inverted.

In terms of the even and odd $\phi$-dependent coefficients, the surface current, (9) and (10), can be written as

$$
\begin{aligned}
& K_{\phi}(\phi, z)=\frac{1}{\pi \sin v} \sum_{p=0}^{\infty} \cos p v \\
& \cdot \sum_{n=0}^{\infty}\left(K_{\phi n}^{(+), p} \cos n \phi+i K_{\phi n}^{(-), p} \sin n \phi\right)
\end{aligned}
$$

$K_{z}(\phi, z)=\frac{1}{\pi} \sum_{p=0}^{\infty} \sin (p+1) v$

$$
\cdot \sum_{n=0}^{\infty}\left(K_{z n}^{(+), p} \cos n \phi+i K_{z n}^{(-), p} \sin n \phi\right) .
$$

\section{ThE FAR FIELD}

The scattered far field from the cylinder can be expressed in terms of the expansion coefficients $K_{z n}^{( \pm), p}$ and $K_{\phi n}^{( \pm), p}$ obtained by solving (15)-(18). From (2) -(4), the far-field components in the spherical coordinate $(r, \theta, \phi)$ are

$$
E_{r}(r, \theta, \phi)=0
$$

$$
\begin{aligned}
& -\frac{2 i}{l_{1} l_{2} \zeta_{0} G(\mathbf{r})} E_{\theta}(r, \theta, \phi) \\
& =-\sum_{n=0}^{\infty} \sum_{p=0}^{\infty} i^{-(n+p)} \frac{(p+1) \sin \theta}{l_{1} \cos \theta} \\
& \cdot J_{p+1}\left(l_{1} \cos \theta\right) J_{n}\left(l_{2} \sin \theta\right)
\end{aligned}
$$

$$
\begin{aligned}
& \cdot\left(K_{z n}^{(+), p} \cos n \phi+i K_{z n}^{(-), p} \sin n \phi\right) \\
& +\sum_{n=1}^{\infty} \sum_{p=0}^{\infty} i^{-(n+p)} \frac{n \cos \theta}{l_{2} \sin \theta} J_{p}\left(l_{1} \cos \theta\right) J_{n}\left(l_{2} \sin \theta\right) \\
\cdot & \left(K_{\phi n}^{(-), p} \cos n \phi+i K_{\phi n}^{(+), p} \sin n \phi\right) \\
& -\frac{2 i}{l_{1} l_{2} \zeta_{0} G(\mathbf{r})} E_{\phi}(r, \theta, \phi) \\
= & \sum_{n=0}^{\infty} \sum_{p=0}^{\infty} i^{-(n+p)} J_{p}\left(l_{1} \cos \theta\right) J_{n}^{\prime}\left(l_{2} \sin \theta\right) \\
& \cdot\left(-K_{\phi n}^{(-), p} \sin n \phi+i K_{\phi n}^{(+), p} \cos n \phi\right) .
\end{aligned}
$$

Two interesting special cases in which the above expressions are simplified are the far fields on the $x-y$ plane and along the $z$-axis. On the $x-y$ plane bisecting the cylinder, $\theta=$ $\pi / 2$ and only the $p=0$ components are involved. In the cylindrical coordinate $(\rho, \phi, z=0)$, the fields are

$$
-\frac{2 i}{l_{1} l_{2} \zeta_{0} G(\mathrm{r})} E_{z}(\rho, \phi, 0)=\frac{1}{2} \sum_{n=0}^{\infty} i^{-n} J_{n}\left(l_{2}\right)
$$$$
\cdot\left(K_{z n}^{(+), 0} \cos n \phi+i K_{z n}^{(-), 0} \sin n \phi\right)
$$

$$
\begin{aligned}
&-\frac{2 i}{l_{1} l_{2} \zeta_{0} G(\mathrm{r})} E_{\phi}(\rho, \phi, 0)=\sum_{n=0}^{\infty} i^{-n} J_{n}^{\prime}\left(l_{2}\right) \\
& \cdot\left(-K_{\phi n}^{(-), 0} \sin n \phi+i K_{\phi n}^{(+), 0} \cos n \phi\right) .
\end{aligned}
$$

Along the $z$-axis, $\theta=0$ or $\pi$ and only the components with $n$ $=1$ contribute to the scattered field. In the rectangular coordinate,

$$
\begin{gathered}
E_{z}(0,0, z)=0 \\
-\frac{2 i}{l_{1} l_{2} \zeta_{0} G(\mathbf{r})} E_{x}(0,0, z)=-\frac{i}{2} \sum_{p=0}^{\infty} i^{\mp p} J_{p}\left(l_{1}\right) K_{\phi 1}^{(-), p} \\
-\frac{2 i}{l_{1} l_{2} \zeta_{0} G(\mathbf{r})} E_{y}(0,0, z)=\frac{1}{2} \sum_{p=0}^{\infty} i^{\mp p} J_{p}\left(l_{1}\right) K_{\phi 1}^{(+), p}
\end{gathered}
$$

where the minus sign applies when $z>0$ and the plus sign applies when $z<0$. 


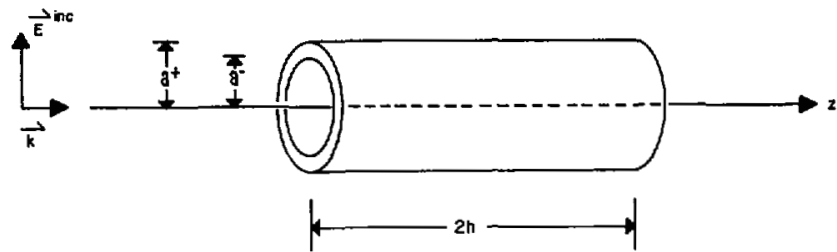

Fig. 3. Experimental configuration.

\section{Comparison with Measured Data}

Backscattering cross sections of two sets of cylinders whose length-to-diameter ratios equal 4 and 6 , respectively, have been measured. A schematic experimental configuration is shown in Fig. 3. Each set consists of four cylinders of different sizes to comprise the data points shown as circles in Figs. 4 and 5. The inner radius $a^{-}$of the cylinder is chosen as the radius $a$ of the idealized cylinder of zero wall thickness used in theoretical computation [12], [13], [14].

Assume that a linearly polarized plane wave given by

$$
\mathbf{E}^{i}=\hat{\mathbf{y}} e^{i l_{1} z}
$$

is incident on the cylinder. On the surface of the cylinder,

$$
\begin{gathered}
E_{z}^{i}(\phi, \cos v)=0 \\
E_{\phi}^{i}(\phi, \cos v)=\cos \phi e^{i l_{1} \cos v .}
\end{gathered}
$$

Thus,

$$
\begin{gathered}
E_{\phi, 1}^{(+), p}=\frac{4(p+1)}{l_{1}^{2} l_{2} \zeta_{0}} i^{(p+1)} J_{p+1}\left(l_{1}\right), \quad p=0,1, \cdots \\
E_{\phi, n}^{( \pm), p}=0, \quad \text { for } n \neq 1,
\end{gathered}
$$

and only the $K_{\phi 1}^{(+), p}$ and $K_{z \mathrm{l}}^{(-), p}$ components exist.

The backscattered $\mathbf{E}$-field has only the $y$-component, and the ratio of the cross section to the projected area of the cylinder $\sigma_{0}=\pi a^{2}$ is

$$
\begin{aligned}
\frac{\sigma}{\sigma_{0}} & =4 \pi \lim _{z \rightarrow-\infty}\left|\frac{1}{k G(\mathbf{r})} E_{y}(0,0, z)\right|^{2} \\
& =\frac{1}{l_{2}^{2}}\left|\sum_{p=0}^{\infty} i^{p} J_{p}\left(l_{1}\right)\left(\frac{l_{1} l_{2} \zeta_{0}}{-2 i} K_{\phi 1}^{(+), p}\right)\right|^{2}
\end{aligned}
$$

which is dependent on $l_{1}$ and $l_{2}$ only.

Cross sections are computed for cylinders having $l_{1} / l_{2}$ values of 4 or 6 with $l_{2}$ varying from 0.875 (not included) to 3.125 at 256 evenly spaced values. Terms of the current expansion coefficients of up to $p=15$ at the highest frequency are retained. The sets of linear equations are stable under inversion. ' The results are plotted as the solid curves in Figs. 4 and 5. Agreement with measured data is evident.

\footnotetext{
' Computations up to $n=8$ have been carried out to provide bistatically scattered fields from nonaxially incident waves. The sets of linear equations are stable under inversion for all these cases. Results will be published when measured data are available.
}

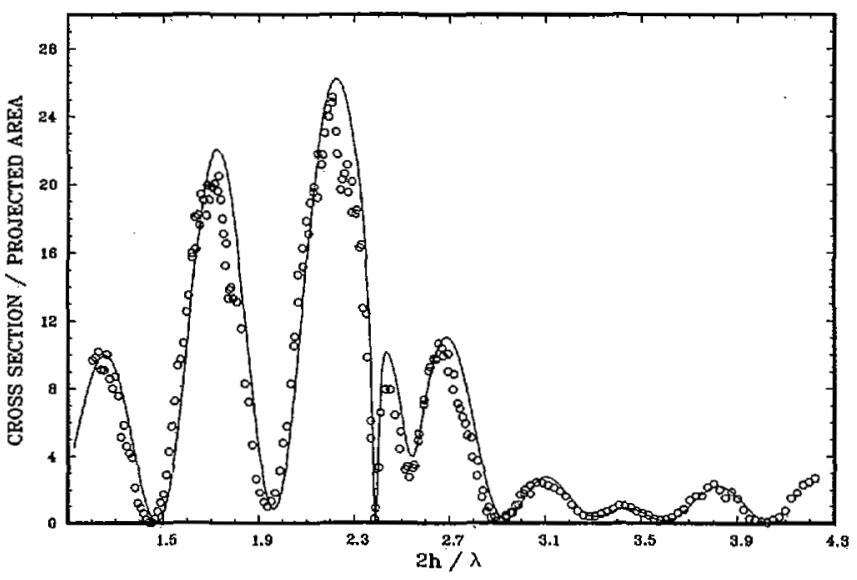

Fig. 4. Backscattering cross section along the axis of a tubular cylinder with $h / a^{-}=4$, theory (solid curve) and experiment (circles).

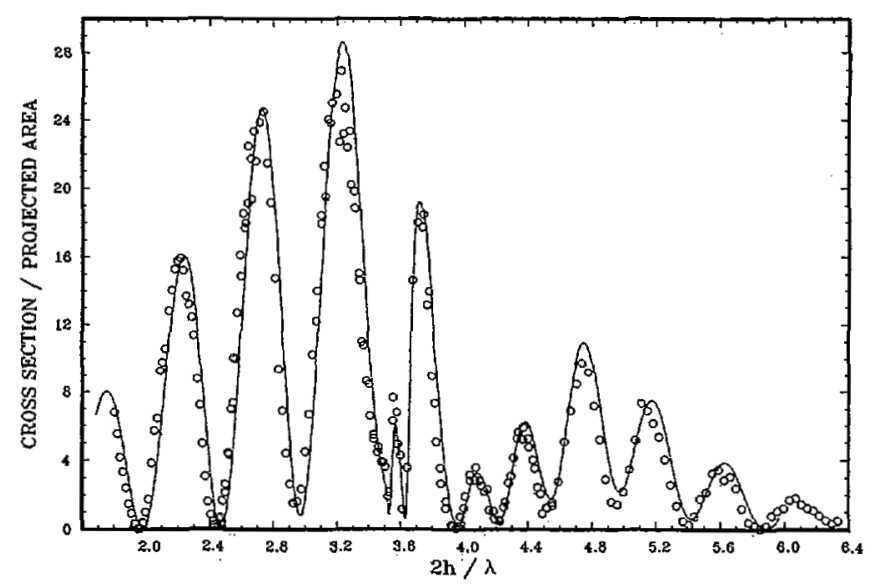

Fig. 5. Backscattering cross section along the axis of a tubular cylinder with $h / a^{-}=5$, theory (solid curve) and experiment (circles).

\section{Concluding Remarks}

Surface current on the finite tubular cylindrical structure has been expanded over the Chebyshev polynomials in a form that the edge condition is satisfied term by term. Systems of linear equations are obtained which determine these expansion coefficients and thus the surface current under external excitation. This formulation applies to both the cylinder case and the loop case and to both scattering problems and antenna problems when the antenna is driven evenly outside and inside the cylinder [5].

This formulation is different from the Hallen type solution [2], [3], [5], [15] in that (8) is not formally integrated and there is no integration constant to be determined. It is different from other techniques in that all the coefficients, $M_{z z n}^{p, q}$ etc., of the linear systems of (15)-(18) are known analytically and no numerical integration is required for their evaluation.

Some special cases are being investigated: when $l_{2} \ll 1$ and $l_{2} \ll l_{1}$, the evaluation of the coefficients in (15)-(18) reduces to a single series sum. This is the case applicable to the linear antennas. When $l_{1} \ll 1$ and $l_{1} \ll l_{2}$, the case of an electrically thin loop obtains. Closed-form expressions can be derived. Results of these studies will be reported elsewhere. 


\section{APPENDIX I}

$$
G_{n}^{p, q} \text { FOR } l_{1} \geq l_{2}, n \geq 0
$$

For $l_{1} \geq l_{2}, G_{n}^{p, q}$ can be written, with $p+q=2 s$ and $|p-q|=2 d$, as

$G_{n}^{p, q}=\frac{i}{2 \pi} \sum_{r=n}^{\infty} \frac{(-1)^{p+n+r} l_{2}^{2 r} \Gamma\left(r+\frac{1}{2}\right)}{\Gamma(r+n+1) \Gamma(r-n+1)} \sum_{m=s}^{\infty} \frac{(-1)^{m} l_{1}^{2 m} \Gamma^{2}\left(m+\frac{1}{2}\right) \Gamma(m+1)}{\Gamma(m+r+3 / 2) \Gamma(m+s+1) \Gamma(m+d+1) \Gamma(m-s+1) \Gamma(m-d+1)}$

$-\frac{1}{2 \pi} \sum_{r=n}^{\infty} \frac{(-1)^{p+n+r} l_{2}^{2 r} \Gamma\left(r+\frac{1}{2}\right)}{\Gamma(r+n+1) \Gamma(r-n+1)} \sum_{m=0}^{\infty} \frac{(-1)^{m} l_{1}^{2 m+1} \Gamma^{2}(m+1) \Gamma(m+3 / 2)}{\Gamma(m+r+2) \Gamma(m+s+3 / 2) \Gamma(m+d+3 / 2) \Gamma(m-s+3 / 2) \Gamma(m-d+3 / 2)}$

$+\frac{1}{4 \pi^{2} l_{1}} \sum_{r=n}^{\infty} \frac{(-1)^{n+r}\left(l_{2} / l_{1}\right)^{2 r} \Gamma\left(r+\frac{1}{2}\right)}{\Gamma(r+n+1) \Gamma(r-n+1)} \sum_{m=0}^{r} \frac{l_{1}^{2(r-m)} \Gamma\left(m+s+\frac{1}{2}\right) \Gamma\left(m+d+\frac{1}{2}\right)}{\Gamma(r-m+1) \Gamma\left(m+\frac{1}{2}\right) \Gamma^{2}(m+1) \Gamma\left(s-m+\frac{1}{2}\right) \Gamma\left(d-m+\frac{1}{2}\right)}$

$\cdot\left\{\left(s_{1}\left(l_{1} / l_{2} ; r, n ; m, s, d\right)\right)^{2}+s_{2}(r, n ; m, s, d)\right\}$

$+\frac{1}{2 \pi^{2} l_{1}} \sum_{r=0}^{n-1} \frac{\left(l_{2} / l_{1}\right)^{2 r} \Gamma\left(r+\frac{1}{2}\right) \Gamma(n-r)}{\Gamma(r+n+1)} \sum_{m=0}^{r} \frac{l_{1}^{2(r-m)} \Gamma\left(m+s+\frac{1}{2}\right) \Gamma\left(m+d+\frac{1}{2}\right)}{\Gamma(r-m+1) \Gamma\left(m+\frac{1}{2}\right) \Gamma^{2}(m+1) \Gamma\left(s-m+\frac{1}{2}\right) \Gamma\left(d-m+\frac{1}{2}\right)}$

$\cdot s_{1}\left(l_{1} / l_{2} ; r, n ; m, s, d\right)$

where

$$
\begin{aligned}
& s_{1}(x ; r, n ; m, s, d) \\
& =2 \ln (x)+\psi(r+n+1)+\psi\left(\left|n-r-\frac{1}{2}\right|+\frac{1}{2}\right)-\psi\left(r+\frac{1}{2}\right)+2 \psi(m+1)+\psi\left(m+\frac{1}{2}\right) \\
& \quad-\psi\left(m+s+\frac{1}{2}\right)-\psi\left(m-s+\frac{1}{2}\right)-\psi\left(m+d+\frac{1}{2}\right)-\psi\left(m-d+\frac{1}{2}\right)
\end{aligned}
$$

$s_{2}(r, n ; m, s, d)$

$$
\begin{aligned}
= & \pi^{2}+\psi^{\prime}\left(r+\frac{1}{2}\right)-\psi^{\prime}(r+n+1)-\psi^{\prime}(r-n+1)-2 \psi^{\prime}(m+1)-\psi^{\prime}\left(m+\frac{1}{2}\right) \\
& -\psi^{\prime}\left(s-m+\frac{1}{2}\right)+\psi^{\prime}\left(s+m+\frac{1}{2}\right)-\psi^{\prime}\left(d-m+\frac{1}{2}\right)+\psi^{\prime}\left(d+m+\frac{1}{2}\right) .
\end{aligned}
$$

$\psi$ is the logarithmic derivative of the gamma function.

The first two double series sums converge rapidly. The fourth sum, being finite, can be carried out easily. The third sum is a power series in $\left(l_{2} / l_{1}\right)^{2}$ whose coefficients are functions of $l_{1}$ and $\ln \left(l_{2} / l_{1}\right)$. When $l_{2} / l_{1}$ approaches 1 , the convergence is slow. Convergence acceleration by Euler-Abel transformation has been adopted which provides accurate results.

APPENDIX II

$$
G_{n}^{p, q} \text { FOR } l_{1} \leq l_{2}, n \geq 0
$$

For $l_{1} \leq l_{2}, G_{n}^{p, q}$ can be written, with $p+q=2 s$ and $|p-q|=2 d$, as 


$$
\begin{aligned}
& G_{n}^{p, q}=\frac{i}{2 \pi} \sum_{m=s}^{\infty} \frac{(-1)^{p+n+m} l_{1}^{2 m} \Gamma(m+1) \Gamma^{2}\left(m+\frac{1}{2}\right)}{\Gamma(m+s+1) \Gamma(m+d+1) \Gamma(m-s+1) \Gamma(m-d+1)} \sum_{r=n}^{\infty} \frac{(-1)^{r} l_{2}^{2 r} \Gamma\left(r+\frac{1}{2}\right)}{\Gamma(r+m+3 / 2) \Gamma(r+n+1) \Gamma(r-n+1)} \\
& -\frac{1}{2 \pi} \sum_{m=s}^{\infty} \frac{(-1)^{p+n+m} l_{1}^{2 m} \Gamma(m+1) \Gamma^{2}\left(m+\frac{1}{2}\right)}{\Gamma(m+s+1) \Gamma(m+d+1) \Gamma(m-s+1) \Gamma(m-d+1)} \sum_{r=0}^{\infty} \frac{(-1)^{r} l_{2}^{2 r} \Gamma(r+1)}{\Gamma(r+m+2) \Gamma(r+n+3 / 2) \Gamma(r-n+3 / 2)} \\
& +\frac{1}{2 \pi^{2} l_{2}} \sum_{m=s}^{\infty} \frac{(-1)^{p+m}\left(l_{1} / l_{2}\right)^{2 m} \Gamma(m+1) \Gamma^{2}\left(m+\frac{1}{2}\right)}{\Gamma(m+s+1) \Gamma(m+d+1) \Gamma(m-s+1) \Gamma(m-d+1)} \sum_{r=0}^{m} \frac{(-1)^{r} l_{2}^{2(m-r)} \Gamma\left(r+n+\frac{1}{2}\right)}{\Gamma(m-r+1) \Gamma(r+1) \Gamma\left(n-r+\frac{1}{2}\right)} \\
& \cdot s_{3}\left(l_{2} / l_{1} ; r, n ; m, s, d\right) \\
& +\frac{(-1)^{d}}{2 \pi^{2} l_{2}} \sum_{m=d}^{s-1} \frac{\left(l_{1} / l_{2}\right)^{2 m} \Gamma(m+1) \Gamma^{2}\left(m+\frac{1}{2}\right) \Gamma(s-m)}{\Gamma(m+s+1) \Gamma(m+d+1) \Gamma(m-d+1)} \sum_{r=0}^{m} \frac{(-1) r l_{2}^{2(m-r)} \Gamma\left(r+n+\frac{1}{2}\right)}{\Gamma(m-r+1) \Gamma(r+1) \Gamma\left(n-r+\frac{1}{2}\right)}
\end{aligned}
$$

where

$$
\begin{aligned}
s_{3}(x ; r, n ; m, s, d) & \\
= & 2 \ln (x)+\psi(r+1)-\psi\left(n+r+\frac{1}{2}\right)-\psi\left(n-r+\frac{1}{2}\right)-2 \psi\left(m+\frac{1}{2}\right)-\psi(m+1) \\
& +\psi(m+s+1)+\psi(m-s+1)+\psi(m+d+1)+\psi(m-d+1) .
\end{aligned}
$$

The same remarks for the case $l_{1} \geq l_{2}$ apply except that, in this case, the third double series sum is a power series in $\left(l_{2} /\right.$ $\left.l_{1}\right)^{2}$.

\section{REFERENCES}

[1] T. T. Wu, "Theory of the dipole antenna and the two-wire transmission line," J. Math. Phys., vol. 2, pp. 550-574, 1961.

[2] R. H. Duncan and F. A. Hinchey, "Cylindrical antenna theory," $J$. Res. Nat. Bur. Stand, vol. 64D, pp. 569-584, 1960.

[3] H.-M. Lee, "Electromagnetic scattering of a loop above a half-space: theory and experiment," Ph.D. dissertation, Harvard Univ., 1981.

[4] R. W. P. King and T. T. Wu, "The thick tubular transmitting antenna," Radio Sci., vol. 2, pp. 1061-1065, 1967.

[5] D. C. Chang, "On the electrically thick cylindrical antenna," Radio Sci., vol. 2, pp. 1043-1060, 1967.

[6] R. W. P. King, B. Sandler, T. T. Wu, R. W. Burton, C. C. Kao and L. C. Shen, "Surface currents and charges on an electrically thick conducting tube in an E-polarized, normally incident, plane-wave field, 1, theory," Radio Sci., vol. 11, pp. 687-699, 1976.

[7] T. W. Johnson and D. L. Moffatt, "Electromagnetic scattering by open circular waveguides," Radio Sci., vol. 17, pp. 1547-1556, 1982.

[8] R. W. P. King and T. T. Wu, The Scattering and Diffraction of Woves. Cambridge, MA: Harvard Univ. Press, 1959.

[9] H.-M. Lee, "Double series expansion of the Green's function for a perfectly conducting tubular cylinder of finite length," Radio Sci., vol. 18 , pp. $48-56,1983$.

[10] J. A. Stratton and L. J. Chu, "Diffraction theory of electromagnetic waves," Phys. Rev., vol. 56, pp. 99-107, 1939.
[11] R. Mittra and S. W. Lee, Analytic Techniques in the Theory of Guided Waves. New York: MacMillan, 1971.

[12] H.-M. Lee, D. Geller, B. Haklay, A. Setiawan, and G. P. Chung, "Back scattering cross section along the axis of a tubular cylinder of finite length," Proc. ISAP '85, vol. III, pp. 815-818, Aug. 1985, Kyoto, Japan.

[13] H.-M. Lee, D. Geller, B. Haklay, and G. P. Chung, " $H_{11}$ waveguide mode and back scattering cross section along the axis of a thin walled tubular cylinder of finite length," Proc. Inst. Elec. Eng., vol. 133, pt. H, pp. 77-78, 1986.

[14] G.-P. Chung, "Effects of waveguide modes on the scattering of a tubular cylinder," M.s. thesis, Naval Postgrad. School, 1985.

[15] C. C. Kao, "Three-dimensional electromagnetic scattering from a circular tube of finite length," J. Appl. Phys., vol. 40, pp. 4732$4740,1969$.

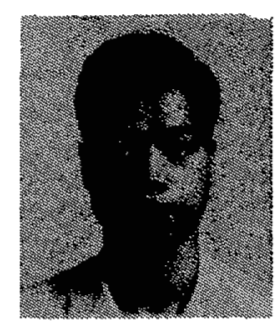

Hung-Mou Lee was born in Taipei, Taiwan, in 1949. He received the B.S. degree from the National Chiao-Tung University, Hsin-Chu. Taiwan in 1970 and the M.A. and Ph.D. degrees in physics from Harvard University, Cambridge, MA, in 1975 and 1981, respectively.

He is an Associate Professor of Electrical and Computer Engineering at the Naval Postgraduate School, Monterey, CA, where he has taught and carried out research in electromagnetic theory and radar systems since 1982 . 